Miller, Lance

[58]

\section{Silencing of Wnt signaling and activation of multiple metabolic pathways in response to cell proliferation stimulated by thyroid hormone}

\author{
Lance Miller 1,2 , Kyung Soo Park ${ }^{3}$, Qingbin Guo ${ }^{1,2}$, \\ Nawal Alkharouf ${ }^{4}$, Renae Malek ${ }^{5}$, Norman Lee ${ }^{5}$, Edison Liu ${ }^{1}$ \\ \& Sheue-yann Cheng ${ }^{2}$ \\ ${ }^{1}$ Department of Cancer and Cell Biology, Medicine Branch, Division of Clinical \\ Sciences, National Cancer Institute, National Institutes of Health, Bethesda, \\ Maryland 20892, USA \\ ${ }^{2}$ Advanced Technology Center, Department of Cancer and Cell Biology, National \\ Cancer Institute, Gaithersburg, Maryland 20877, USA \\ ${ }^{3}$ Laboratory of Molecular Biology, Medicine Branch, Division of Clinical Sciences, \\ National Cancer Institute, National Institutes of Health, Bethesda, Maryland \\ 20892, USA \\ ${ }^{4}$ School of Computational Sciences, George Mason University, Fairfax, Virginia \\ 22030, USA \\ ${ }^{5}$ Department of Functional Genomics, Institute for Genomic Research, Rockville, \\ Maryland 20850, USA
}

To investigate the transcriptional program underscoring cell proliferation induced by thyroid hormone (T3), we used complementary DNA mircroarrays to survey the temporal expression profiles of 4,400 genes in a classical T3 model. Of 352 responsive genes identified, $77 \%$ had not previously been reported to be transcriptionally or functionally modulated by $\mathrm{T} 3$, thereby increasing the number of reported T3 genes by approximately $250 \%$. Partitioning the genes into functional classes revealed the activation of multiple pathways (including those for glucose metabolism, biosynthesis, transcriptional regulation, protein degradation and stress response and detoxification) in T3-induced cell proliferation. Clustering the genes by temporal expression patterns and expression timing provided further insight into the dynamics of $\mathrm{T} 3$ response pathways. Of particular significance was the finding that $\mathrm{T} 3$ rapidly repressed the expression of a host of key regulators of the tumor-promoting Wnt signaling pathway and the transcriptional activity of the $\beta$-catenin-TCF complex. Furthermore, activation of Wnt signaling led to inhibition of T3-mediated cell proliferation. These results indicate that cell proliferation induced by thyroid hormone is accompanied by a complex, coordinated transcriptional reprogramming of many genes in different pathways and that early silencing of the Wnt pathway is critical to this event.

Monni, Outi

\section{7q23 amplification in breast cancer leads to overexpression of multiple genes}

Outi Monni', Maarit Barlund², Juha Kononen', Guido Sauter ${ }^{3}$, Olli Kallioniemi ${ }^{1}$ \& Anne Kallioniemi ${ }^{1}$

${ }^{1}$ Cancer Genetics Branch, National Human Genome Research Institute, National Institutes of Health, Bethesda, Maryland, USA

${ }^{2}$ Institute of Medical Technology, University of Tampere and Tampere University Hospital, Tampere, Finland

${ }^{3}$ Institute of Pathology, University of Basel, Basel, Switzerland

Comparative genomic hybridization studies indicate that the $17 \mathrm{q} 23$ region is one of the most common amplification sites in breast cancer and therefore that it may harbor genes playing an important role in tumor progression. We have used a combination of molecular, genomic and microarray technologies to identify systematically target genes for this amplicon. We constructed a genomic contig for the 17 q23 region (approximately 4 megabases) and used these genomic clones to map amplicon boundaries in 5 breast cancer cell lines and 372 primary breast cancers; 17 known genes and 26 expressed sequence tags were localized to the contig. Analysis of the genomic sequence of BAC clones from this region revealed 34 additional Unigene clusters and 43 individual expressed sequence tags. To perform a complete expression survey, we constructed a complementary DNA microarray containing 116 clones from our contig and 522 clones from other regions on chromosome 17. Microarray analysis on eight breast cancer cell lines revealed a limited number of consistently overexpressed genes, including $S 6 K, R A D 51 C$ and $A P P B P 2$, and several unknown expressed sequence tags. The $S 6 \mathrm{~K}$ coding for a regulator of the G1-S transition showed high-level overexpression by northern and western blot analysis as well as increased functional activity when amplified in breast cancer cell lines. S6K was amplified in $8.8 \%$ and overexpressed in $15 \%$ of primary breast tumors and showed a significant association with poor prognosis $(P<0.002)$. Comprehensive analysis of the $17 \mathrm{q} 23$ amplicon revealed genes that may have oncogenic potential and may contribute to the more aggressive clinical course in breast cancer patients with 17q23-amplified tumors.

Montpetit, Alexandre

$[60]$

\section{A detailed transcript map of the human chromosome 12p12.3 tumor suppressor locus: the usefulness of an integrative approach}

\section{Alexandre Montpetit, Nathalie Trudel \& Daniel Sinnett}

Charles-Bruneau Cancer Centre, Division of Hemato-Oncology, Research Center, Ste-Justine Hospital, Montreal, Canada

Allellic loss of the chromosome $12 \mathrm{p}$ is a frequent event in childhood acute lymphoblastic leukemia. This region was also found deleted in several other hematological malignancies as well as a variety of solid tumors suggesting the presence of a tumor suppressor gene. The chromosomal region containing this suppressor locus was narrowed down to a an interval of approximately $750 \mathrm{~kb}$ delimited by D12S98 and D12S358. As we could not identify a known candidate gene, we initiated the construction of a detailed transcription map focusing on a contig of 4 overlapping BACs. Towards this goal we applied a strategy integrating several complementary approaches: (1) Computer-based data mining tools applied to the existing genomic sequence ( $750 \mathrm{~kb})$ derived form the BAC contig; (2) Exon amplification and EST resources to identify putative cDNAs; (3) Determination of general expression pattern by RT-PCR and Northern blotting; (4) Comparative genomic analysis with distant vertebrate species such as Fugu rubripes and Tetraodon nigroviridis. This transcript mapping strategy has identified 32 potential transcription units, including 2 known genes, 5 new genes, 9 Unigene entries and 16 other EST clusters. The region contains also 5 pseudogenes. This map should facilitate subsequent effort to characterize the candidate genes. This study is a good illustration of how the integration of genome-based approaches facilitates the identification of genes in large interval. 